\title{
REPERFUSION INJURY WITH RENAL REPERCUSSION LEADING TO AMPUTATION AS A COMPLIGATION OF RADIAL ARTERY CANNULATION
}

\author{
S. Gaspar Santos $\left(^{*}\right)$, F. Rosa ${ }^{(*)}$ \\ ${ }^{(*)}$ Rigshospitalet - Glostrup - Copenhagen (Denmark), ${ }^{(*)}$ Ospedale Santa Maria degli Angeli - Pordenone (Italy)
}

\section{Backgroundz}

Arterial line placement has a major complication rate below $1 \%$. Ischemic injury can occur in patients with impaired perfusion or hemodynamic instability.

Reperfusion injury is a life-threatening situation and this is, to the best of our knowledge, the first report of its occurrence after radial artery cannulation.

\section{Case Report:}

68-year-old woman was admitted to the ICU due to cardiogenic shock. Relevant treatment, including vasopressors, was started.

An arterial line was placed in left radial artery, but stopped working shortly after and was subsequently removed. Both the left forearm and hand became pale and cold. Doppler was performed and showed no flow in both ulnar and radial arteries, as well as a weak pulse in the left brachial artery. The vascular surgery team was contacted and opted for conservative treatment, due to the patient's critical condition.

After 12 hours, the patient developed left forearm pain, as well as anesthesia and paresis of the I and II left fingers. A new Doppler showed a permeable ulnar artery, but no flow in the radial artery starting from mid-forearm.

Restoration of circulation was, at this point, attempted with sympaticolysis (axillary block), administration of salicylate and arterial vasodilation (nimodipin). This resulted in a better perfused hand, warm fingertips, but persistent ischemic area in the forearm.

In the course of the following 12 hours, the patient developed severe pain, the ischemic area increased and muscle rigidity in the entire left forearm and hand developed. Doppler showed no flow in both ulnar and radial arteries. Concomitantly, a rapid deterioration of renal function was observed.

After a multidisciplinary team discussion, left midarm amputation was performed due to the presence of severe ischemia and reperfusion injury.

The patient was released from the ICU on the 6th day.

\section{Discussion:}

Female sex, hypotension and use of vasopressors are all known risk factors for the development of hand ischemia after radial artery cannulation. ${ }^{1}$

Reperfusion injury is a risk to be considered in the treatment of ischemia after radial artery cannulation.

In general, muscle appears to be tolerant to ischemia for up to 4 hours, nerve changes remain reversible up to 8 hours, fat up to 13 hours, skin up to 24 hours, and bone up to 4 days at normothermia. An inflammatory response in the ischemic tissue follows reperfusion, due to the release of breakdown products (myoglobin, potassium, lactate, microthrombi, etc.) In case of severe ischemia, a systemic inflammatory response following reperfusion can occur, resulting in a diffuse vascular permeability problem and third space fluid loss, which can in turn lead to hypovolemia and renal hypoperfusion. Attempts to reperfuse the ischemic area after the golden period of 4-6 hours can produce both local and systemic complications. ${ }^{2}$

In our case, the attempt to restore circulation after more than $12 \mathrm{~h}$ in a patient that already had risk factors for renal dysfunction, led to both worsening of ischemia and reperfusion injury with renal repercussion.

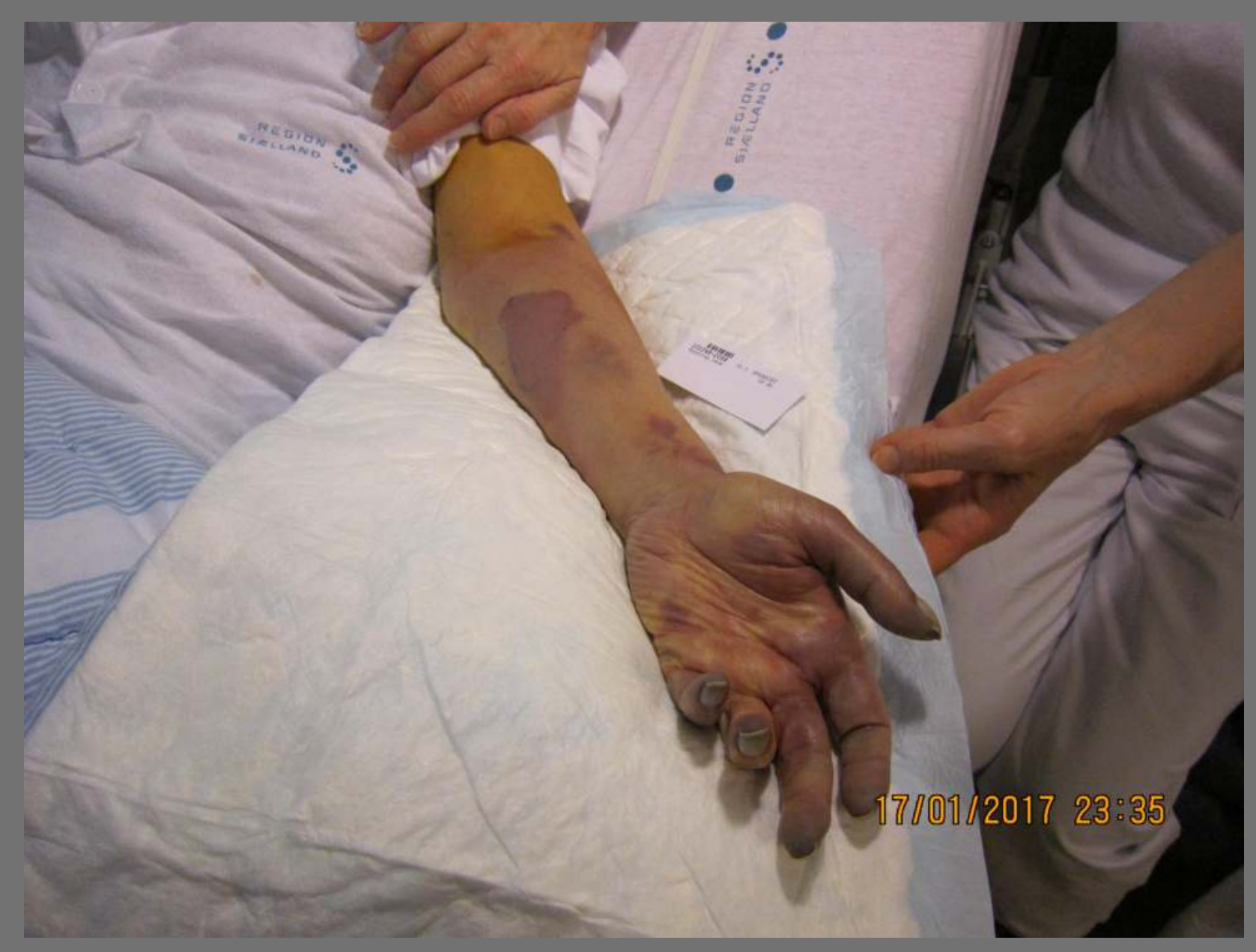

${ }^{1}$ Chir Main. 2014 Sep;33(4):299-302 2 Cardiovasc Surg. 2002 Dec;10(6):620-30 